\title{
Descontaminação de drogas vegetais empregando irradiação gama e óxido de etileno: aspectos microbianos e químicos
}

\author{
Lucilia Cristina Satomi, Renata Rabelo Soriani, Terezinha de Jesus Andreoli Pinto*1 \\ Departamento de Farmácia, Faculdade de Ciências Farmacêuticas, Universidade de São Paulo
}

*Correspondência:

T. J. A. Pinto

Departamento de Farmácia, Faculdade

de Ciências Farmacêuticas

Universidade de São Paulo

Avenida Professor Lineu Prestes, 580-

Bloco13A, Cidade Universitária

05508900 São Paulo-SP

Email: tjapinto@usp.br

\begin{abstract}
A avaliação da qualidade sanitária de drogas vegetais, bem como a utilização de métodos de descontaminação constituem importantes etapas no que se refere ao aspecto de segurança ao consumidor, principalmente pelo fato de serem usualmente consumidas por pessoas debilitadas, por vezes imunodeprimidas. No Brasil a RDC 48/2004 menciona a pesquisa de contaminantes microbiológicos bem como o estudo da eficácia dos agentes descontaminantes. Os objetivos do presente trabalho foram avaliar os efeitos da fumigação com óxido de etileno e da irradiação gama sobre a carga microbiana, bem como determinar alterações nos constituintes químicos em amostras de Ginkgo biloba e Paulinia cupana H.B.K. (guaraná). A carga microbiana inicial foi, em

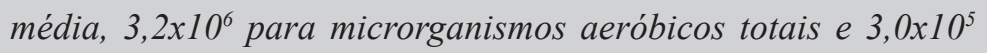
para fungos. Ambos os métodos de descontaminação apresentaramse eficazes na redução da carga microbiana. As análises por cromatografia líquida de alta eficiência revelaram ausência de alterações significativas nos teores de glicosídeos flavonoídicos e cafeina, respectivamente para ginkgo e guaraná.
\end{abstract}

Unitermos

- Drogas vegetais

- Qualidade microbiana

- Óxido de etileno

- Irradiação gama

- Glicosideos flavonoídicos

- Cafeína

\section{INTRODUÇÃO}

Apesar dos grandes avanços da indústria farmacêutica, o interesse em terapias alternativas tem aumentado, especialmente aquelas que empregam drogas vegetais. A Organização Mundial da Saúde estima que $65-80 \%$ da população que vive em países em desenvolvimento, depende essencialmente de plantas medicinais como tratamento primário à saúde (Calixto, 2000). No Brasil 20\% da população é responsável por $63 \%$ do consumo dos medicamentos disponíveis. O restante encontra nos produtos de origem natural, especialmente as plantas medicinais, a única fonte de recursos terapêuticos (Simões, 2000).
À medida que cresce o consumo destas drogas, aumenta a responsabilidade das agências regulatórias e dos fabricantes a fim de que se assegure a qualidade e eficácia terapêutica.

As drogas vegetais, normalmente, apresentam elevada carga microbiana, quer seja saprófita ou patogênica (Satomi, 2003; Soriani, 2004). Os microrganismos contaminantes, normalmente, são provenientes do solo, da água e do ar. Contaminação secundária pode ainda ocorrer devido às práticas de agricultura, ao armazenamento $\mathrm{e}$ ao processamento destas drogas (Katusin-Razem, 2001).

Com o objetivo de eliminar os efeitos decorrentes da biocarga presente nas drogas vegetais, agentes 
descontaminantes, de natureza física ou química, têm sido empregados. A utilização de tais procedimentos de descontaminação, prevista na mais recente regulamentação da Agência Nacional de Vigilância Sanitária, a RDC $\mathrm{n}^{\circ} 48$ de 16 de março de 2004, requer estudos relacionados à estabilidade dos princípios ativos após exposição ao agente selecionado (Brasil, 2004).

A exposição ao gás óxido de etileno é um método eficaz. Entretanto, devido ao risco relacionado aos efeitos carcinogênicos e mutagênicos dos resíduos, seu uso tem sido restringido ou, até mesmo, proibido em certos países, como aqueles da União Européia (Fang; Wu, 1998).

Uma alternativa que tem sido intensivamente adotada é a descontaminação com irradiação gama devido a sua aplicabilidade na ausência de água e temperatura elevada (Aziz et al., 1997; Fang, Wu, 1998; Migdal, Owczarczyk, 1998; Kim, Yook; Byun, 2000; Owczarczyk, Migdal, Kadzia, 2000), merecendo, entretanto, cuidados quanto à verificação de incompatibilidades e realização de estudos de estabilidade, tendo em vista a possibilidade de ocorrerem alterações químicas e sensoriais indesejáveis.

Os objetivos do presente trabalho foram comparar os efeitos da fumigação com óxido de etileno e da irradiação gama sobre a carga microbiana de duas espécies de drogas vegetais: ginkgo (Ginkgo biloba L.) e guaraná (Paullinia cupana H.B.K.), bem como determinar pos- síveis alterações provocadas pelos agentes descontaminantes sobre os teores de seus princípios ativos.

\section{MATERIAL E MÉTODOS}

\section{Material}

As drogas vegetais ginkgo (Ginkgo biloba L.) e guaraná (Paullinia cupana H.B.K.), provenientes de três fornecedores locais (A, B e C), foram acondicionadas em: (i) embalagens filmes plásticos (polietileno-poliester) providas de "air vents" em Tyvek ${ }^{\circledR}$ para as amostras submetidas à fumigação (1 kg/embalagem); e (ii) embalagens de polietileno para as amostras submetidas à radiação (1 kg/embalagem).

\section{Métodos}

\section{Fumigação com óxido de etileno}

Foi adotado o método de esterilização conforme desenvolvido por McDonald (1972), segundo o Gráfico 1. O equipamento utilizado foi o esterilizador Sercon modelo HS E 39/40 HETO 3000. Os tempos de exposição aplicados foram de 30 e 60 minutos, sendo que para cada tempo foram realizadas três réplicas. Após termino da exposição, as drogas vegetais foram mantidas sob aeração forçada por 14 dias consecutivos.

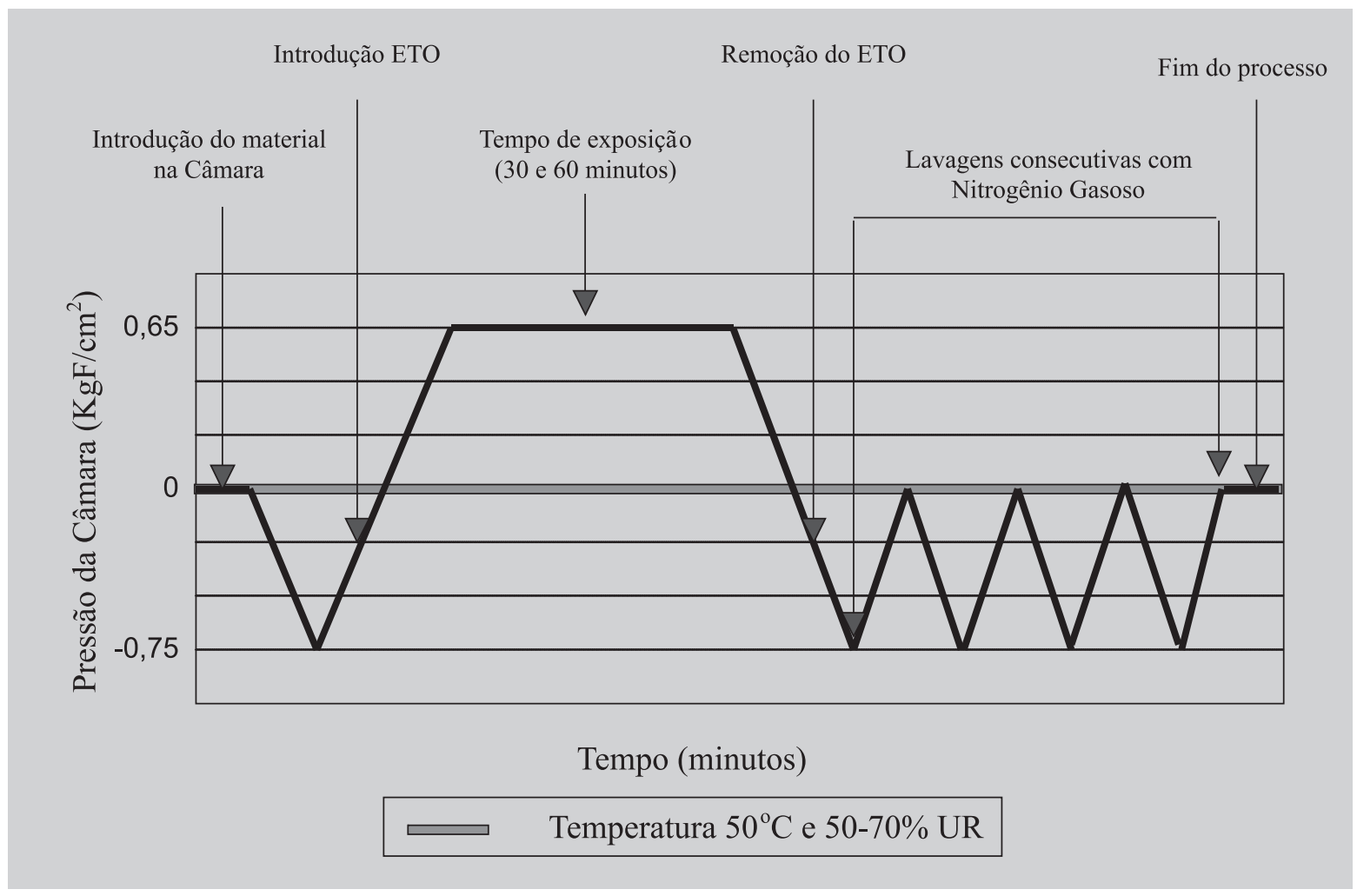

GRÁFICO 1 - Ciclo de Descontaminação por Óxido de Etileno. 
Irradiação

A irradiação das amostras foi realizada no canal experimental de um irradiador gama de grande porte, tipo JS 7500 da Empresa Brasileira de Radiações (EMBRARAD). A monitoração da dose alvo foi realizada através da disposição de dosímetros de metacrilato de polimetila (Perspex ${ }^{\circledR}$ ). Para cada dose, foram realizadas três réplicas. As doses médias absorvidas foram 5,5, 11,4 e $17,8 \mathrm{kGy}$.

\section{Análises microbianas}

As contagens de microrganismos aeróbicos totais e fungos (bolores e leveduras) foram realizadas empregando a técnica de semeadura em profundidade, respectivamente em ágar caseína-soja e ágar Sabouraud dextrose, conforme descrito na Farmacopéia Americana 25 (United States Pharmacopeia, 2002). A pesquisa de microrganismos potencialmente patogênicos Escherichia coli, Pseudomonas aeruginosa, Staphylococcus aureus e Salmonella sp., foi realizada de acordo com Farmacopéia Brasileira $4^{\mathrm{a}}$ edição (1988).

\section{Análises químicas}

A análise de cada espécie vegetal foi efetuada com amostra do fornecedor C. Como etapa preliminar ao doseamento dos marcadores, foram determinados os parâmetros de validação: linearidade, exatidão e repetibilidade, conforme RDC 84 de 2002 da Agência Nacional de Vigilância Sanitária (Brasil, 2002).

Para as amostras de ginkgo, a extração e a quantificação de glicosídeos flavonoídicos foram realizadas de acordo com a Farmacopéia Americana (2002), utilizando sistema de cromatografia líquida de alta eficiência Thermo Separation Products (software PC100) equi- pado com detector por Ultravioleta a $370 \mathrm{~nm}$ e coluna de octadecilsilano $(4,6 \mathrm{~mm} \times 12,5 \mathrm{~cm})$.

O teor de cafeína nas amostras de guaraná foi determinado segundo método descrito por Salvadori et al. (1994), empregando o mesmo sistema de cromatografia líquida, entretanto com detector a $267 \mathrm{~nm}$ e coluna de octadecilsilano de $4,0 \mathrm{~mm} \times 10,0 \mathrm{~cm}$.

Os resultados obtidos foram submetidos à análise estatística.

\section{RESULTADOS E DISCUSSÃO}

Os resultados referentes à contaminação microbiana inicial revelaram elevados níveis de microrganismos aeróbicos totais e fungos em todas as amostras testadas (Tabelas I e II). Este panorama corroborou com estudos anteriores e correspondeu à expectativa, uma vez que o material de origem natural não havia sido previamente submetido a qualquer processamento com a finalidade de reduzir a biocarga (Negretti, 1983; Martins, 2001; KatusinRazem, 2001).

Após 30 e 60 minutos de exposição ao óxido de etileno evidenciou-se a eficácia do método (Tabela I). Com relação à contagem de microrganismos aeróbicos totais, após 30 minutos de exposição, as amostras de ginkgo apresentaram menor redução logarítmica ( 0 a 2 ciclos) quando comparadas com as amostras de guaraná (4 a 5 ciclos). A redução da carga fúngica foi de aproximadamente 3 ciclos logarítmicos para todas as drogas. Entretanto, após 60 minutos de exposição ao gás, observou-se redução ao nível de sensibilidade do método, ou seja, $<10 \mathrm{UFC} / \mathrm{g}$.

No quesito detecção de patogênicos específicos nas amostras antes do ciclo de exposição, foram encontrados

TABELA I - Carga microbiana em drogas vegetais antes e após fumigação por óxido de etileno

\begin{tabular}{|c|c|c|c|c|c|c|c|}
\hline \multirow{4}{*}{ Droga vegetal } & \multirow{4}{*}{ Fornecedor } & \multicolumn{6}{|c|}{ Grupo microbiano (UFC/g) $)^{\mathrm{a}, \mathrm{b}, \mathrm{c}}$} \\
\hline & & \multirow{2}{*}{\multicolumn{3}{|c|}{$\frac{\text { Microrganismos aeróbicos totais }}{\text { Tempo de exposição (minutos) }}$}} & \multicolumn{3}{|c|}{ Fungos } \\
\hline & & & & & Tempo d & xposiçã & nutos) \\
\hline & & $\mathbf{0}$ & 30 & 60 & $\mathbf{0}$ & 30 & 60 \\
\hline \multirow[t]{3}{*}{ Ginkgo } & $\mathrm{A}$ & $1,0 \times 10^{4}$ & $8,0 \times 10^{3}$ & $<10$ & $4,4 \times 10^{3}$ & $1,2 \times 10^{2}$ & $<10$ \\
\hline & $\mathrm{B}$ & $5,4 \times 10^{3}$ & $1,2 \times 10^{2}$ & $<10$ & $5,8 \times 10^{2}$ & $<10$ & $<10$ \\
\hline & $\mathrm{C}$ & $1,0 \times 10^{4}$ & $1,0 \times 10^{3}$ & $<10$ & $1,2 \times 10^{3}$ & $<10$ & $<10$ \\
\hline \multirow[t]{3}{*}{ Guaraná } & $\mathrm{A}$ & $5,9 \times 10^{6}$ & $7,5 \times 10^{2}$ & $<10$ & $2,3 \times 10^{4}$ & $3,4 \times 10^{2}$ & $<10$ \\
\hline & $\mathrm{B}$ & $4,5 \times 10^{4}$ & $<10$ & $<10$ & $5,9 \times 10^{3}$ & $1,2 \times 10$ & $<10$ \\
\hline & $\mathrm{C}$ & $7,8 \times 10^{5}$ & $5,0 \times 10$ & $<10$ & $2,1 \times 10^{5}$ & $7,8 \times 10^{2}$ & $<10$ \\
\hline
\end{tabular}

${ }^{\mathrm{a}} \mathrm{UFC}=$ unidades formadoras de colônia. ${ }^{\mathrm{b}}$ Média de três réplicas. ${ }^{\mathrm{c}}(<10)=$ ausência de crescimento nas três réplicas 
resultados presuntivos para $P$. aeruginosa nas amostras de ginkgo dos fornecedores $\mathrm{B}$ e $\mathrm{C}$ e em todas as amostras de guaraná. E. coli foi encontrada nas amostras de guaraná dos três fornecedores. Entretanto não foram detectados os microrganismos pesquisados após o menor dos tempos de exposição. Estes resultados não surpreendem por já haver sido comprovada a alta sensibilidade desses microorganismos ao óxido de etileno (Michael, Stumbo, 1970).

Após irradiação com dose média de 5,5 kGy, observou-se a não uniformidade entre as amostras na redução da carga de microrganismos aeróbicos totais, que variou de 1 a 5 ciclos logarítmicos (Tabela II). Adose média de 11,4 kGy reduziu a contagem destes microrganismos a níveis da ordem de 10. Com relação ao efeito da irradiação sobre a carga fúngica (Tabela II), constatou-se que a menor dose média absorvida $(5,5 \mathrm{kGy})$ foi suficiente para reduzir a contagem inicial para valores aceitáveis em todas as amostras.

Os resultados encontrados são coerentes, pois, de acordo com dados encontrados em literatura, doses de 3 a $10 \mathrm{kGy}$ podem reduzir o número de microrganismos aeróbicos totais a níveis menores que $10^{3} \mathrm{a} 10^{4} \mathrm{UFC} / \mathrm{g}$, e doses entre 16 e $20 \mathrm{kGy}$ a menos que $10 \mathrm{UFC/g}$ (Farkas,
1988). Aziz et al. (1997) constataram que dose de $5 \mathrm{kGy}$ foi eficaz para eliminação de bolores em plantas medicinais.

No que se refere à pesquisa de microrganismos patogênicos nas amostras não irradiadas, houve indicativos de $P$. aeruginosa nas amostras de guaraná provenientes dos fornecedores A e B, e de E. coli no guaraná do fornecedor B. Após irradiação, não foram encontrados os microrganismos pesquisados.

Com relação à determinação de glicosídeos flavonoídicos em ginkgo, o teor mínimo especificado pela $25^{\mathrm{a}}$ edição da Farmacopéia Americana é de 0,8\% (United States Pharmacopeia, 2002). Entretanto, em sua penúltima edição a especificação tornou-se menos rígida e o limite mínimo passou a ser de $0,5 \%$ (United States Pharmacopeia, 2003). Os resultados obtidos para as duas amostras, anteriormente à descontaminação (Tabelas III e IV), foram próximos entre si e acima da especificação. As investigações realizadas por Sticher (1993) revelaram concentrações de 0,5 a $1 \%$ de glicosídeos flavonoídicos em amostras comerciais de folhas secas de ginkgo e não foram observadas diferenças significativas entre os resultados obtidos nos diferentes meses de coleta (junho a novembro).

TABELA II - Carga microbiana em drogas vegetais antes e após irradiação

\begin{tabular}{|c|c|c|c|c|c|c|c|c|c|}
\hline \multirow{4}{*}{ Droga vegetal } & \multirow{4}{*}{ Fornecedor } & \multicolumn{8}{|c|}{ Grupo microbiano (UFC/g) $)^{\mathrm{a}, \mathrm{b}, \mathrm{c}}$} \\
\hline & & \multirow{2}{*}{\multicolumn{4}{|c|}{$\frac{\text { Microrganismos aeróbicos totais }}{\text { Dose de radiação }(\mathrm{kGy})}$}} & \multicolumn{4}{|c|}{ Fungos } \\
\hline & & & & & & Dos & de rad & $\operatorname{ação~(kC~}$ & \\
\hline & & $\mathbf{0}$ & 5,5 & 11,4 & 17,8 & $\mathbf{0}$ & 5,5 & 11,4 & 17,8 \\
\hline \multirow[t]{3}{*}{ Ginkgo } & $\mathrm{A}$ & $1,8 \times 10^{4}$ & $2,0 \times 10^{2}$ & 2 & $<10$ & $2,5 \times 10^{2}$ & 2 & 2 & 2 \\
\hline & $\mathrm{B}$ & $9,8 \times 10^{6}$ & $8,8 \times 10$ & 1 & $<10$ & $2,3 \times 10^{3}$ & 3 & 3 & $<10$ \\
\hline & $\mathrm{C}$ & $1,2 \times 10^{4}$ & $2,7 \times 10$ & 2 & 1 & $6,0 \times 10^{2}$ & 5 & 3 & 3 \\
\hline \multirow[t]{3}{*}{ Guaraná } & $\mathrm{A}$ & $1,4 \times 10^{7}$ & $5,2 \times 10^{3}$ & $2,2 \times 10$ & $<10$ & $1,6 \times 10^{4}$ & 3 & 1 & 1 \\
\hline & $\mathrm{B}$ & $7,3 \times 10^{6}$ & $1,6 \times 10^{3}$ & $2,0 \times 10$ & 1 & $3,3 \times 10^{6}$ & $3,3 \times 10$ & $1,2 \times 10$ & 3 \\
\hline & $\mathrm{C}$ & $2,3 \times 10^{3}$ & $2,3 \times 10^{2}$ & 5 & 1 & $6,1 \times 10^{4}$ & 1 & 3 & 1 \\
\hline
\end{tabular}

${ }^{a} \mathrm{UFC}=$ unidades formadoras de colônia. ${ }^{\mathrm{b}}$ Média de três réplicas. ${ }^{\mathrm{c}}(<10)=$ ausência de crescimento nas três réplicas.

TABELA III - Teores dos marcadores nas drogas vegetais antes a após fumigação por óxido de etileno

\begin{tabular}{ccc}
\hline Tempo de exposição (min) & \multicolumn{2}{c}{ Droga vegetal / marcador ${ }^{\mathbf{a}}$} \\
\cline { 2 - 3 } & Ginkgo / glicosídeos flavonoídicos (\%)* & Guaraná / Cafeína (\%) $^{*}$ \\
\hline 0 & 1,21 & 3,83 \\
30 & 1,25 & 3,93 \\
60 & 1,23 & 3,55 \\
\hline
\end{tabular}

\footnotetext{
${ }^{a}$ Média de três determinações.
} 
TABELA IV - Teores dos marcadores nas drogas vegetais antes a após irradiação

\begin{tabular}{ccc}
\hline Dose de radiação (kGy) & \multicolumn{2}{c}{ Droga vegetal / marcadora } \\
\cline { 2 - 3 } & Ginkgo / glicosídeos flavonoídicos (\%)* & Guaraná / Cafeína (\%) $^{\text {(\%) }}$ \\
\hline 0 & 1,12 & 4,03 \\
5,5 & 1,07 & 4,17 \\
11,4 & 1,02 & 4,12 \\
17,8 & 1,02 & 4,23 \\
\hline
\end{tabular}

${ }^{a}$ Média de três determinações.

Os teores de cafeína nas amostras de guaraná não descontaminadas foram superiores a 3,25\%, concentração preconizada pela Farmacopéia Brasileira (Farmacopéia Brasileira, 1988). Resultados semelhantes foram obtidos por Carlson e Thompson (1998) que analisaram 4 amostras comerciais de guaraná com teores de 3,8 a 4,1\% de cafeína.

Com relação às amostras submetidas aos processos de descontaminação, as variações encontradas antes e após submissão aos agentes (Tabelas III e IV) não foram estatisticamente significativas e, portanto, foram consideradas como erro analítico.

\section{CONCLUSÃO}

Com base nos resultados anteriormente apresentados, ambos os métodos podem ser considerados eficazes na redução da carga microbiana para as drogas vegetais avaliadas, além de não causarem alterações no teor dos marcadores adotados. Entretanto, vale ressaltar que devido aos riscos associados aos resíduos tóxicos de óxido de etileno, o método que emprega este agente requer pelo menos 14 dias de aeração forçada para eliminação total dos mesmos. Deve, ainda, ser ressaltado que, em situações experimentais distintas, caracterizadas por diferentes espécies vegetais, biocargas e, portanto, condições de tratamento também diferentes, deverá ser confirmada a estabilidade química após os procedimentos.

\section{ABSTRACT \\ Decontamination of vegetal drugs using gama irradiation and ethylene oxide: microbial and chemical aspects}

The sanitary quality of vegetal drugs, as well as the use of decontamination methods are important steps towards the consumer safety, mainly due to the fact that these products are usually used by sick and with weak immunocompromised persons. In Brazil, the RDC 48/
2004 mention the contamination research as well as the efficacy of the decontaminated product. The purpose of this work is to evaluate the effects of ethylene oxide fumigation and gamma irradiation on the microbial burden and some chemical constituents of ginkgo and guarana. The initial microbial burden was $3,2 \times 10^{6}$, on average, for total aerobic microorganisms and 3,0x105 for fungi. Both methods proved to be effective in the microbial burden reduction. The analyses using high performance liquid chromatography revealed the absence of significant alterations in the flavonol glycosides and caffein contents, respectively for ginkgo and guarana.

UNITERMS: Vegetal drugs. Microbial quality. Ethylene oxide. Gama irradiation. Flavonol glycosides. Caffein.

\section{AGRADECIMENTOS}

À FAPESP (Fundação de Amparo à Pesquisa do Estado de São Paulo) pelo auxílio financeiro.

\section{REFERÊNCIAS BIBLIOGRÁFICAS}

AZIZ, N.H. et al. Effect of gamma radiation on the survival of fungal and actinomycetal florae contaminating medicinal plants. Appl. Radiat. Isot., Oxford, v.48, n.1, p.71-76, 1997.

BRASIL. Ministério da Saúde. Agência Nacional de Vigilância Sanitária. Diretoria Colegiada. Resolução n. 84 de 19 de março de 2002. Lex Coletânea de Legislação e Jurisprudência, São Paulo, v. 66, p. 1005-1014, 2002.

BRASIL. Ministério da Saúde. Secretaria Nacional de Vigilância Sanitária. Diretoria Colegiada. Resolução n. 48 de 16 de março de 2004. Diário Oficial, Brasília, DF, 18 de março de 2004. Seção 1, v.53, p. 39-41. 
BRITISH PHARMACOPOEIA. London: Her Majesty's Stationery Office, 2000. v. 2, p. 130-172.

CALIXTO, J.B. Efficacy, safety, quality control, marketing and regulatory guidelines for herbal medicines (phytotherapeutic agents). Braz. J. Méd. Biol. Res., Ribeirão Preto, v.33, n.2, p.179-189, 2000.

CARLSON, M.; THOMPSON R.D. Liquid chromatographic determination of methylxantines and catechins in herbal preparations containing guaraná. J. AOAC Int., Washington, v.81, n.4, p.691-701, 1998.

FANG X.; WU J. Feasibility of sterilizing traditional medicines by gamma-irradiation. Radiat. Phys. Chem., Oxford, v.52, n.1-6, p.53-58, 1998.

FARKAS, J. Irradiation of dry food ingredients. Florida: CRC Press, Inc., 1988. p.1-76.

FARMACOPÉIA BRASILEIRA. 4.ed. São Paulo: Atheneu São Paulo, 1988. p. v.2.9, v.5.1.6, v.5.1.7.

FISCHER, D.C.H. Contaminação microbiana em medicamentos fitoterápicos sob a forma sólida. São Paulo, 1992. 161p [Tese de Mestrado. Faculdade de Ciências Farmacêuticas. Universidade de São Paulo].

KATUSIN-RAZEM, B.; NOVAK, B.; RAZEM, D. Microbiological decontamination of botanical raw materials and corresponding pharmaceutical products by irradiation. Radiat. Phys. Chem., Oxford, v.62, p.261-275, 2001.

KIM, M.; YOOK, H.; BYUN, M. Effects of gamma irradiation on microbial contamination and extraction yields of Korean medicinal herbs. Radiat. Phys. Chem., Oxford, v.57, p.55-58, 2000.

MARTINS, H.M. et al. Evaluation of microbiological quality of medicinal plants used in natural infusions. Int. J. Food Microbiol., Oxford, v.68, p. 149-153, 2001.

McDONALD, R.L. Method of sterilizing. U.S. Pat. 3.068.064, 1962. Apud. PHILIPS, G.B., MILLER, W.S., eds. Industrial sterilization. Durham: Duke University Press, 1972. p.181-208.

MICHAEL, G.T., STUMBO, C.R. Ethylene oxide sterilization of Salmonella senftenberg and Escherichia coli: death kinetics and mode of action. J. Food Sci., Chicago, v.35, p.631-634, 1970.
MIGDAL, W.; OWCZARCZYK, B. The effect of ionizing radiation on microbiological decontamination of medical herbs and biologically active compounds. Radiat. Phys. Chem., Oxford, v.52, n1-6, p.91-94, 1998.

NEGRETTI, F. Ricerche sulla contaminazione microbica dei prodotti fitoterapici. Boll. Chim. Farm., Milano, v.122, 1983.

OWCZARCZYK, H.B.; MIGDAL, W.; KADZIA, B. The pharmacological activity of medical herbs after microbiological decontamination by irradiation. Radiat. Phys Chem., Poland, v.57, p.331-335, 2000.

SALVADORI, M.C. et al. Determination of xantines by highperformance liquid chromatography and thin-layer chromatography in horse urine after ingestion of guaraná powder. Analyst, London, v.119, p.2701-2703, 1994.

SATOMI, L.C. Estudo da aplicabilidade do processo de descontaminação de drogas vegetais empregando óxido de etileno. São Paulo, 2003. 157 p. [Tese de Mestrado. Faculdade de Ciências Farmacêuticas. Universidade de São Paulo].

SIMÕES, C.M.O., org. Farmacognosia: da planta ao medicamento. 2.ed. Florianópolis: Ed. da UFRGS / Ed. da UFSC, 2000. 821p.

SORIANI, R.R. Irradiação de drogas vegetais: aspectos microbiológicos e químicos. São Paulo, 2004. 129 p. [Tese de Mestrado. Faculdade de Ciências Farmacêuticas. Universidade de São Paulo].

STICHER, O. Quality of Ginkgo preparations. Planta Med. Stuttgart, v.59, p.2-11, 1993.

TOOFANIAN, F.; STEGEMAN, H. Comparative effect of ethylene oxide and gamma irradiation on the chemical, sensory and microbial quality of ginger, cinnamon, fennel and fenugreek. Acta Aliment., Budapest, v.17, n.4, p.271281,1988 .

UNITED STATES PHARMACOPEIA. 25.ed. Rockville, United States Pharmacopeial Convention, 2002. 2569p.

UNITED STATES PHARMACOPEIA. 26.ed. Rockville, United States Pharmacopeial Convention, 2003. 2921p.

Recebido para publicação em 22 de setembro de 2004. Aceito para publicação em 09 de março de 2005. 